\title{
Is the new ASNM intraoperative neuromonitoring supervision "guideline" a trustworthy guideline? A commentary
}

\author{
Stanley A. Skinner ${ }^{1}$ - Elif Ilgaz Aydinlar ${ }^{2}$. Lawrence F. Borges ${ }^{3} \cdot$ Bob S. Carter $^{4} \cdot$ Bradford L. Currier $^{5}$. \\ Vedran Deletis ${ }^{6} \cdot$ Charles Dong $^{7} \cdot$ John Paul Dormans $^{8} \cdot$ Gea Drost $^{9} \cdot$ Isabel Fernandez-Conejero ${ }^{10}$. \\ E. Matthew Hoffman ${ }^{11}$. Robert N. Holdefer ${ }^{12}$. Paulo Andre Teixeira Kimaid ${ }^{13}$. Antoun Koht ${ }^{14} \cdot K_{\text {Karl F. Kothbauer }}^{15}$. \\ David B. MacDonald ${ }^{16}$. John J. McAuliffe $\mathrm{III}^{17}$. David E. Morledge ${ }^{18}$. Susan H. Morris ${ }^{19}$. Jonathan Norton ${ }^{20}$. \\ Klaus Novak ${ }^{21}$. Kyung Seok Park ${ }^{22}$. Joseph H. Perra ${ }^{23}$. Julian Prell ${ }^{24}$ • David M. Rippe ${ }^{25}$. Francesco Sala ${ }^{26}$. \\ Daniel M. Schwartz ${ }^{27} \cdot$ Martín J. Segura $^{28} \cdot$ Kathleen Seidel $^{29} \cdot$ Christoph Seubert $^{30} \cdot$ Mirela V. Simon $^{31}$. \\ Francisco Soto ${ }^{32}$. Jeffrey A. Strommen ${ }^{33,34}$. Andrea Szelenyi ${ }^{35}$. Armando Tello ${ }^{36}$. Sedat Ulkatan ${ }^{37}$. Javier Urriza ${ }^{38}$. \\ Marshall Wilkinson ${ }^{39}$
}

Received: 17 December 2018 / Accepted: 22 December 2018 / Published online: 5 January 2019

(c) The Author(s) 2019, corrected publication 2019

\section{Introduction: provider-centered versus patient-centered IONM supervision}

The new ASNM intraoperative neuromonitoring (IONM) supervision guideline [1] attempts to justify current remote IONM practices. ${ }^{1}$ Contrary to ordinary clinical practice guideline development, it is provider-centered, not patient-centered.

The new guideline could have embraced recent scholarship that indicates the need to provide robust teamwork and medical error avoidance. It could have moved in the direction of improved patient safety and outcomes. Instead, key words are repeated ("communicate," "collaborate," "team") many times, but with no meaningful strategy to achieve their patient safety potential. In fact, within this new guideline, an IONM remote provider's communication with in-room physician peers and co-practitioners is defined as: “... at minimum, direct voice access (via 'land-line' or cellular network) for perioperative communication with the surgical team".

Endorsed by: Peter Newton, M.D.-Scoliosis Research Society (SRS) President; Paul Sponseller, M.D.--SRS President elect; Muharrem Yazici, M.D.-SRS Vice President; Todd Albert, M.D.-SRS Past President, On behalf of the SRS.

Stanley A. Skinner

Stanley.Skinner@allina.com

Extended author information available on the last page of the article

\section{The evidence for optimized intraoperative team communication and decision-making}

Two-thirds of medical errors derive from poor team performance during patient care [2,3]. Such errors are lessened with rigorous adherence to situational awareness and critical language [4]. "Talk among physicians is essential in the negotiation of professional relationships, the distribution of responsibility, the inducement of cooperation, and the assessment of competence [5]." And, "The current weaknesses in communication in the OR may derive from a lack of standardization and team integration... decisions are often made without all relevant team members present, and much communication is consequently reactive and tension provoking [6]." Furthermore, scholars increasingly recommend using all means to reduce errors and emphasize team familiarity as a major element contributing to patient safety [7,8].

Improved outcomes with IONM depend on timely and appropriate surgeon responses to IONM alerts [9-11]. The assertion that an unseen, distant, and often unknown interpreter can effectively discuss the implications of an IONM alarm during crisis management should be questioned. The

\footnotetext{
${ }^{1}$ By remote IONM, we mean the dominant US model of IONM in which the supervising neurophysiologist is often unfamiliar with, distant from, and unavailable to in-room colleagues (either personally or virtually) and is situationally unaware. Local hub to satellite "remote" IONM of less complex cases and among familiar team members can be fraught with diminished situational awareness, but is not the focus of this commentary.
} 
added expertise and counsel provided by the supervising IONM provider allows the surgeon to weigh the risks and benefits of the available options. It is highly unlikely that an off-site provider, available only by phone, could provide the same value in these high-risk and stressful situations.

Based on a systematic review of operating room teamwork, communication, and safety, "[Operating room] culture improvement appears to be associated with... positive effects, including better patient outcomes" [12]; support for this conclusion appears in a separate review [13]. Furthermore, the World Health Organization's Guidelines for Safe Surgery stipulate all the above prerequisites, again based on systematic review [14].

Clearly, there is substantial evidence that the most direct flow of information between all intraoperative team members is an important factor to ensure quality patient care. Thus, it is reasonable to assume that the limited communication inherent to remote IONM may adversely affect quality and patient outcomes. Since the new guideline provides no evidence that this is not the case, it should not recommend a voice access minimum standard.

\section{Remote IONM is not the virtual patient care practiced in teleStroke or teleICU}

Because remote IONM is telecommunication based, a pertinent review of virtual medical care would have been helpful. However, the new guideline reflects unawareness of the benefits of enriched telecommunication versus the harms of poor telecommunication. For example, controlled trials comparing phone-based to audiovisually enriched teleStroke care have shown that patient outcomes are poorer using phone based communication [15-17]. Also, a major teleICU controlled study revealed that patient outcomes are similar when audiovisually enriched virtual care is compared to personal care [18].

Unfortunately, current remote IONM practices exemplify poor telecommunication. These essential questions were raised in 2010 [19]: "How can an individual outside the OR interpret fluctuating neuromonitoring data and develop explanations of cause? How it is possible to verify an appropriate level of vigilance on the part of the remotely connected professional, particularly if multiple cases are being monitored simultaneously?" To date, no answers have been forthcoming.

But the new guideline further asserts that with a phone connection and internet waveform display, the supervising neurophysiologist effectively: "Communicates and collaborates with other members of the patient care team"; "Evaluates IONM data in the context of the procedure"; "Evaluates and interprets data obtained from topographical/neuro-navigation studies"; and "determines if changes are related to iatrogenic injury, anesthetic effects, physiological variables, patient positioning, technical factors, or a combination of these".

One may extend the concerns unanswered since 2010. How is it possible to achieve these duties without personal or virtual situational awareness? How is it possible to make credible recommendations during a situationally unaware phone call between practitioners who are barely or not at all known to each other?

The new guideline does stipulate: "It is further recognized that cases of greater complexity may require personal attendance in the operating room." What are those cases? For any IONM case of any complexity, which of the duties listed above can be routinely addressed by a phone connection?

\section{Remote IONM and patient care}

The new guideline states that IONM supervision "constitutes a patient care activity", but its actual language does not support this role: "Guideline authors recommend direct or indirect interaction with the patient to the extent possible. This can be accomplished through multiple pathways, including a collaboration with the IONM-T [technologist]". The Centers for Medicare \& Medicaid Services (CMS) stipulate that telemedicine services are "provided to the patient in 'real time' by the telemedicine practitioner, similar to the actions of an on-site practitioner when called in by a patient's attending physician to see the patient" $[15,20]$; there is no intermediary nurse or technologist.

Remote providers do not engage in either direct or virtual patient care by the definitions of CMS or by best practices within tele-ICU or teleStroke care. Therefore, it would have been more forthright to simply state that the remote provider interprets waveforms and e-chats with the technician/ technologist or telephones other staff as needed. At best, that is what really happens during most remote IONM. This non-patient care role has also been defined by CMS to cover tele-radiology, for example [20]. Potentially effective tools to achieve virtual IONM patient care are readily available, but are not stipulated in the new guideline.

\section{Stakeholder engagement}

There are multiple stakeholders in the dialogue occurring during IONM. The new guideline fails to account for the perspective of surgeons, anesthesiologists, or patients about the limitations of the remote monitoring approach. The new guideline does not include support for the proposed recommendations from the surgeon or the anesthesiology community. This feedback should have been sought and broadly 
developed before proceeding. A consensus approach would develop true guidelines as to the specific nature of the remote IONM activity addressing such issues as: telephone/ chat only versus audiovisually enhanced IONM, distractibility issues including number of cases permitted to be simultaneously monitored, and the physical location of the remote monitoring physician. Just as surgeons and anesthesiologists have immediate physical availability requirements in the OR environment, supervising IONM providers, as key members of the operative team, should be held to similarly high standards.

\section{Guideline or position statement?}

The original 2014 ASNM supervision guideline [21] was drafted before the Institute of Medicine's 2011 "Clinical Practice Guidelines We Can Trust" [22] became a widely accepted standard. In retrospect, the 2014 document was actually a position statement. Certainly, since 2014, Institute of Medicine standards should have been followed. These stipulate that, "To be trustworthy, guidelines should be based on a systematic review of the existing evidence", and that "Clinical practice guidelines fundamentally rest on appraisal of the quality of relevant evidence, comparison of the benefits and harms of particular clinical recommendations, and value judgments regarding the importance of specific benefits and harms." While the new guideline states that ASNM "periodically publishes Clinical Practice Guidelines consistent with the Institute of Medicine," it fails to meet any of these standards. It may be a position statement, but it is not a clinical practice guideline.

The new guideline offers an unsound justification for an update and "over-write" of the original 2014 supervision guideline: “ $\ldots$ that [2014] document has undergone review and revision to accommodate broad inter- and intra-societal feedback." In fact, updating guidelines, under the Institute of Medicine's report, is a formal task: "Changes in evidence, the values placed on evidence, the resources available for health care, and improvements in current performance are all possible reasons for updating clinical guidelines [22, 23]." Neither majority societal opinion nor the fraction of clinicians currently meeting a systematized evidential criterion creates a legitimate basis for a guideline update or replacement.

\section{Conclusion}

The new IONM supervision "guideline" asserts that routine phone-based communication with the surgeon and other members of the operating room team meets a minimum expectation, but offers no supportive empirical evidence.
Crucial surgeon and anesthesiology stakeholders were not consulted. It does not conform to Institute of Medicine standards; it does not provide systematized evidence, benefit/harms analysis, or disclosure(s) of potential conflicts of interest with the remote monitoring industry. Therefore, by Institute of Medicine criteria, the article is not a guideline.

Unfortunately, the literature demonstrating that optimized operating room collaboration avoids surgical errors has been ignored. The telemedicine literature recommending enhanced audiovisual connectivity in high risk environments like the ICU and during teleStroke care has been excluded. The new guideline may be interpreted as a license for maintenance of the status quo, thereby inhibiting the adoption of new technologies that have the potential to elevate the quality of remote monitoring.

Although "communication" and "collaboration" and "patient care" are rhetorically supported, no effective mechanisms are described to realize these patient-centered goals. Effective communication within multidisciplinary teams does not start and end with a case. It is increasingly acquired over years of collaborative work.

The new "guideline" appears to be chiefly aimed at protecting the business model of the remote monitoring industry. Surgeons, hospitals, payers, and the broader IONM community may wish to assess the implications of its many flawed premises.

Acknowledgements Stanley A Skinner, M.D.: Director, Intraoperative Neurophysiology Department, Abbott Northwestern Hospital Minneapolis, MN, USA; Endorsed by: Peter Newton, M.D.: Scoliosis Research Society (SRS) President, Paul Sponseller, M.D.: SRS President elect Muharrem Yazici, M.D.: SRS Vice President Todd Albert, M.D.: SRS Past President, On behalf of the SRS; Elif Ilgaz Aydinlar, M.D.: Treasurer, International Society of Intraoperative Neurophysiology, Acibadem University School of Medicine, Department of Neurology, Istanbul, Turkey; Lawrence F. Borges, M.D.: Associate Professor of Neurosurgery, Massachusetts General Hospital, Harvard Medical School, Boston, MA; Bob S. Carter, M.D., Ph.D.: William and Elizabeth Sweet Professor of Neurosurgery, Harvard Medical School, Head of the Department of Neurosurgery, Massachusetts General Hospital, Boston, MA; Bradford L. Currier, M.D.: Professor of Orthopedics and Neurosurgery, Consultant, Department of Orthopedics, Director, Spine Surgery Fellowship, Mayo Clinic, Rochester, MN, USA; Vedran Deletis, M.D., Ph.D.: Past President International Society of Intraoperative Neurophysiology, Albert Einstein College of Medicine, New York, NY, USA; Charles Dong, Ph.D.: Founding member Canadian Association of Neurophysiological Monitoring, Clinical Associate Professor, Division of Neurosurgery, Department of Surgery, University of British Columbia, Vancouver, British Columbia, Canada; John Paul Dormans, M.D.: Chief, Pediatric Orthopedic Surgery, Riley Hospital for Children, Garceau Professor of Orthopedic Surgery, Indiana University School of Medicine, Indianapolis, IN, USA; Gea Drost, M.D., Ph.D.: Secretary International Society of Intraoperative Neurophysiology, Department of Neurosurgery/Neurology, University Medical Center Groningen, Groningen, The Netherlands; Isabel Fernandez-Conejero, M.D.: President elect International Society of Intraoperative Neurophysiology, Department of Intraoperative Neurophysiology, Hospital Universitari de Bellvitge, L'Hospitalet de Llobregat, Barcelona, Spain; E. Matthew Hoffman, D.O., Ph.D.: Assistant Professor, Department of Neurology, 
Mayo Clinic, Rochester, MN, USA; Robert N. Holdefer, Ph.D.: Associate Professor, Department of Rehabilitation Medicine, University of Washington, Harborview Medical Center, Seattle, WA, USA; Paulo Andre Teixeira Kimaid, M.D., Ph.D.: President Latin American Chapter International Federation Clinical Neurophysiology, Past President Brazilian Society of Clinical Neurophysiology, Chairman, Intraoperative Neuromonitoring Section, Department of Neurosurgery, Federal University of São Paulo, São Paulo, Brazil; Antoun Koht, M.D.: Past President American Society Neurophysiological Monitoring, Professor of Anesthesiology, Neurological surgery and Neurology, Chief, neurosurgical anesthesia, Northwestern University, Feinberg School of Medicine, Chicago, IL, USA; Karl F. Kothbauer, M.D.: Chief, Division of Neurosurgery, Luzerner Kantonsspital, Associate Professor, University of Basel, CH-6000 Luzern, Switzerland; David B. MacDonald, M.D., FRCP(C), ABCN: Department of Neurosciences, Section of Neurophysiology, King Faisal Specialist Hospital \& Research center, MBC 76, PO Box 3354, Riyadh, 11211, Saudi Arabia; John J. McAuliffe III, M.D., MBA: Past President American Society Neurophysiological Monitoring, Professor and Anesthesiologist-in-Chief, Department of Anesthesia, Cincinnati Children's Hospital, The University of Cincinnati, Cincinnati, Ohio, USA; David E Morledge, Ph.D., CCC-A, DABNM, FASNM, Past President American Society Neurophysiological Monitoring, Neurostatus Eagle, ID USA'Susan H. Morris, Ph.D.: Past President Canadian Association of Neurophysiological Monitoring, IWK Children's Health Program \& Division of Neurosurgery, Dalhousie University Halifax, NS Canada; Jonathan Norton, Ph.D.: Founding member and Past President Canadian Association Neurophysiological Monitoring, Assistant Professor and Clinical Neurophysiologist, Department of Surgery, University of Saskatchewan, Saskatoon, Saskatchewan, Canada; Klaus Novak, M.D.: Dept. of Neurosurgery, Medical University of Vienna, Vienna, Austria; Kyung Seok Park, M.D., Ph.D.: Delegate by Liaison, International Society of Intraoperative Neurophysiology, Professor, Department of Neurology, Seoul National University Bundang Hospital, Seoul National University College of Medicine, Seongnam, Republic of Korea; Joseph H Perra, M.D.: Twin Cities Spine Center, Minneapolis, MN USA; Julian Prell, M.D., P.D.: Credentialing Committee (Co-Chair) International Society Intraoperative Neurophysiology, Martin Luther University of Halle-Wittenberg, Department of Neurosurgery, Halle (Saale), Germany; David M Rippe, M.D.: Neurophysiology Department, Abbott Northwestern Hospital Minneapolis, MN, USA; Francesco Sala, M.D.: Professor and Chairman, Section of Neurosurgery, Dept. of Neurosciences, Biomedicine and Movement Sciences, University Hospital, Verona, Italy; Daniel M Schwartz, Ph.D.: Founding Member American Society Neurophysiological Monitoring, Founder Surgical Monitoring Associates Springfield, PA USA; Martín J. Segura, M.D., Ph.D.: Past President Argentine Society of EEG and Clinical Neurophysiology, Founding member Special Interest Group Neurophysiological Intraoperative Monitoring, Latin-American, Chapter International Federation of Clinical Neurophysiology, Assistant Professor University of Buenos Aires Medical School, Head Clinical Neurophysiology Unit, Hospital Garrahan, Buenos Aires, Argentina; Kathleen Seidel, M.D.: Attending Physician, Intraoperative Neurophysiology and Neuro-oncological Surgery, Department of Neurosurgery, Inselspital Bern University Hospital, Bern, Switzerland; Christoph Seubert, M.D., Ph.D.: Professor of Anesthesiology and Neurosurgery, Chief, Division of Neuroanesthesia University of Florida, College of Medicine, Gainesville, Florida USA; Mirela V. Simon, M.D., M.Sc.: Associate Professor of Neurology, Harvard Medical School, Director, Intraoperative Neurophysiology Unit, Program Director, IONM Fellowship, Department of Neurology, Massachusetts General Hospital, Boston, MA, USA; Francisco Soto, M.D.: President International Society of Intraoperative Neurophysiology, Neurology Department, Clínica Las Condes, Santiago de Chile; Jeffrey A. Strommen, M.D.: Assistant professor, Department of Physical Medicine and Rehabilitation, Mayo Clinic, Rochester, MN, USA; Neurophysiology Department, Abbott Northwestern Hospital, Minneapolis, MN, USA; Andrea Szelenyi, M.D., P.D.: Prof. Dr. Andrea Szelenyi, Past
President International Society of Intraoperative Neurophysiology, Clinical and Intraoperative Neurophysiology, Department of Neurosurgery, Hospital of University of Munich, Ludwig Maximilians Universitaet Muenchen (LMU), Munich, Germany; Armando Tello, M.D., Ph.D.: Past President Mexican Society of Clinical Neurophysiology, Founding Member IONM Special Interest Group: Latin American Chapter International Federation, Clinical Neurophysiology, Head, Clinical Neurophysiology Department, Hospital Español, Mexico City, Mexico; Sedat Ulkatan, M.D., DABNM, CNIM: Director of Intraoperative Monitoring Service, Mount Sinai West Hospital-New York NY. USA; Javier Urriza, M.D.: Educational Committee Co-Chair International Society of Intraoperative Neurophysiology, Intraoperative Neurophysiology Unit, Clinical Neurophysiology Deptartment, Complejo Hospitalario de Navarra-B, Pamplona-Iruña, Navarra, Spain; Marshall Wilkinson, B.Sc. (Hons), M.Sc., Ph.D.: Current President Canadian Association Neurophysiological Monitoring, Section of Neurosurgery, University of Manitoba, Winnipeg, Manitoba, Canada.

Funding No author received funding for the authorship of this commentary.

\section{Compliance with ethical standards}

Conflict of interest Dr. Skinner receives a patent royalty from Medtronic, Inc. No pertinent conflicts of interest are reported.

Open Access This article is distributed under the terms of the Creative Commons Attribution 4.0 International License (http://creativeco mmons.org/licenses/by/4.0/), which permits use, duplication, adaptation, distribution and reproduction in any medium or format, as long as you give appropriate credit to the original author(s) and the source, provide a link to the Creative Commons license and indicate if changes were made.

\section{References}

1. Gertsch JH, Moreira JJ, Lee GR, et al. J Clin Monit Comput. 2018. https://doi.org/10.1007/s10877-018-0201-9.

2. Kohn LT, Corrigan J, Donaldson MS. To err is Human: building a safer health system. Washington, DC: National Academies Press; 2000.

3. Wilson RM, Runciman WB, Gibberd RW, Harrison BT, Newby L, Hamilton JD. The quality in Australian health care study. Med J Aust. 1995;163:458-71.

4. Leonard M, Graham S, Bonacum D. The human factor: the critical importance of effective teamwork and communication in providing safe care. Qual Saf Health Care. 2004;13:185-90.

5. Lingard L, Reznick R, Espin S, et al. Team communications in the operating room: talk patterns, sites of tension, and implications for novices. Acad Med. 2002;77:232-7.

6. Lingard L, Espin E, Whyte S, et al. Communication failures in the operating room: an observational classification of recurrent types and effects. Qual Saf Health Care. 2004;13:330-4.

7. ElBardissi AW, Sundt TM. Human factors and operating room safety. Surg Clin North Am. 2012;92:21-35.

8. Wiegmann DA, ElBardissi AW, Dearani JA, Daly RC, Sundt TM III. Disruptions in surgical flow and their relationship to surgical errors: an exploratory investigation. Surgery 2007;142:658-65.

9. Croskerry P. From mindless to mindful practice-cognitive bias and clinical decision making. $\mathrm{N}$ Engl J Med. 2013;368(26):2445-8. 
10. Berner ES, Graber ML. Overconfidence as a cause of diagnostic error in medicine. Am J Med. 2008;121(5):S2-S23.

11. Sala F. Intraoperative neurophysiology is here to stay. Child's Nerv Syst. 2010;26(4):413-7.

12. Sacks GD, Shannon EM, Dawes AJ, et al. Teamwork, communication and safety climate: a systematic review of interventions to improve surgical culture. BMJ Qual Saf. 2015;24:458-67.

13. Skinner S, Holdefer R, McAuliffe JJ, Sala F. Medical error avoidance in intraoperative neurophysiological monitoring: the communication imperative. J Clin Neurophysiol. 2017;34(6):477-83.

14. World Health Organization. In: Gawande A, Weiser T, editors. WHO Guidelines for Safe Surgery 2009: safe surgery saves lives. Geneva: WHO Press; 2009.

15. Skinner S. Patient-centered care model in IONM. J Clin Neurophysiol. 2013;30:204-9.

16. Meyer BC, Raman R, Hemmen T, Obler R, Zivin JA, Rao R, Thomas RG, Lyden PD. Efficacy of site-independent telemedicine in the STRokE DOC trial: a randomised, blinded, prospective study. Lancet Neurol. 2008;7(9):787-95.

17. Handschu R, Scibor M, Willaczek B, Nückel M, Heckmann JG, Asshoff D, Belohlavek D, Erbguth F, Schwab S. Telemedicine in acute stroke. J Neurol. 2008;255(11):1792-7.
18. Lilly CM, McLaughlin JM, Zhao H, Baker SP, Cody S, Irwin RS. A multicenter study of ICU telemedicine reengineering of adult critical care. Chest. 2014;145(3):500-7.

19. Dormans JP. Establishing a standard of care for neuromonitoring during spinal deformity surgery. Spine. 2010;35(25):2180-5.

20. U.S. Department of Health and Human Services, Centers for Medicare \& Medicaid Services. Medicare and Medicaid programs: changes affecting hospital and critical access hospital conditions of participation: telemedicine credentialing and privileging (RIN 0938-AQ05). 2011. Available at: http://www.gpo. gov/fdsys/pkg/FR-2011-05-05//html/FR-2011-05-05-FrontMatte r.htm. Accessed 8 Feb 2012.

21. Skinner SA, Cohen BA, Morledge DE, McAuliffe JJ, Hastings JD, Yingling CD, McCaffrey M. Practice guidelines for the supervising professional: intraoperative neurophysiological monitoring. J Clin Monit Comput. 2014;28(2):103-11.

22. Steinberg E, Greenfield S, Wolman DM, Mancher M, Graham $\mathrm{R}$, editors. Clinical practice guidelines we can trust. Washington, DC: National Academies Press; 2011.

23. Shekelle P, Eccles MP, Grimshaw JM, Woolf SH. When should clinical guidelines be updated? BMJ 2001;323(7305):155.

\section{Affiliations}

Stanley A. Skinner ${ }^{1}$ - Elif Ilgaz Aydinlar ${ }^{2}$. Lawrence F. Borges ${ }^{3}$ - Bob S. Carter ${ }^{4}$ - Bradford L. Currier ${ }^{5}$. Vedran Deletis ${ }^{6} \cdot$ Charles Dong $^{7} \cdot$ John Paul Dormans ${ }^{8} \cdot$ Gea Drost $^{9} \cdot$ Isabel Fernandez-Conejero $^{10}$. E. Matthew Hoffman ${ }^{11}$. Robert N. Holdefer ${ }^{12}$. Paulo Andre Teixeira Kimaid ${ }^{13}$. Antoun Koht ${ }^{14}$. Karl F. Kothbauer ${ }^{15}$. David B. MacDonald ${ }^{16}$. John J. McAuliffe $\mathrm{III}^{17}$. David E. Morledge ${ }^{18}$. Susan H. Morris ${ }^{19}$. Jonathan Norton ${ }^{20}$. Klaus Novak ${ }^{21}$. Kyung Seok Park ${ }^{22}$. Joseph H. Perra ${ }^{23}$. Julian Prell ${ }^{24}$ • David M. Rippe ${ }^{25}$. Francesco Sala ${ }^{26}$. Daniel M. Schwartz ${ }^{27} \cdot$ Martín J. Segura $^{28} \cdot$ Kathleen Seidel $^{29} \cdot$ Christoph Seubert $^{30} \cdot$ Mirela V. Simon $^{31}$. Francisco Soto ${ }^{32}$. Jeffrey A. Strommen ${ }^{33,34}$. Andrea Szelenyi ${ }^{35}$. Armando Tello ${ }^{36}$. Sedat Ulkatan ${ }^{37}$. Javier Urriza ${ }^{38}$. Marshall Wilkinson ${ }^{39}$

1 Intraoperative Neurophysiology Department, Abbott Northwestern Hospital, Minneapolis, MN, USA

2 Acibadem University School of Medicine, Department of Neurology, Istanbul, Turkey

3 Massachusetts General Hospital, Harvard Medical School, Boston, MA, USA

4 Department of Neurosurgery, Harvard Medical School, Massachusetts General Hospital, Boston, MA, USA

5 Department of Orthopedics, Mayo Clinic, Rochester, MN, USA

6 Albert Einstein College of Medicine, New York, NY, USA

7 Division of Neurosurgery, Department of Surgery, University of British Columbia, Vancouver, BC, Canada

8 Pediatric Orthopedic Surgery, Riley Hospital for Children, Indiana University School of Medicine, Indianapolis, IN, USA

9 Department of Neurosurgery/Neurology, University Medical Center Groningen, Groningen, The Netherlands

10 Department of Intraoperative Neurophysiology, Hospital Universitari de Bellvitge, L'Hospitalet de Llobregat, Barcelona, Spain

11 Department of Neurology, Mayo Clinic, Rochester, MN, USA
12 Department of Rehabilitation Medicine, University of Washington, Harborview Medical Center, Seattle, WA, USA

13 Department of Neurosurgery, Federal University of São Paulo, São Paulo, Brazil

14 Feinberg School of Medicine, Northwestern University, Chicago, IL, USA

15 Division of Neurosurgery, Luzerner Kantonsspital, University of Basel, 6000 Lucerne, Switzerland

16 Department of Neurosciences, Section of Neurophysiology, King Faisal Specialist Hospital \& Research Center, MBC 76, PO Box 3354, Riyadh 11211, Saudi Arabia

17 Department of Anesthesia, Cincinnati Children's Hospital, The University of Cincinnati, Cincinnati, OH, USA

18 Neurostatus, Eagle, ID, USA

19 IWK Children's Health Program \& Division of Neurosurgery, Dalhousie University, Halifax, NS, Canada

20 Department of Surgery, University of Saskatchewan, Saskatoon, SK, Canada

21 Department of Neurosurgery, Medical University of Vienna, Vienna, Austria 
22 Department of Neurology, Seoul National University Bundang Hospital, Seoul National University College of Medicine, Seongnam, Republic of Korea

23 Twin Cities Spine Center, Minneapolis, MN, USA

24 Department of Neurosurgery, Halle (Saale), Germany

25 Neurophysiology Department, Abbott Northwestern Hospital, Minneapolis, MN, USA

26 Section of Neurosurgery, Department of Neurosciences, Biomedicine and Movement Sciences, University Hospital, Verona, Italy

27 Surgical Monitoring Associates, Springfield, PA, USA

28 Clinical Neurophysiology Unit, Hospital Garrahan, University of Buenos Aires Medical School, Buenos Aires, Argentina

29 Intraoperative Neurophysiology and Neuro-oncological Surgery, Department of Neurosurgery, Inselspital Bern University Hospital, Bern, Switzerland

30 Division of Neuroanesthesia, College of Medicine, University of Florida, Gainesville, FL, USA

31 Intraoperative Neurophysiology Unit, Department of Neurology, Harvard Medical School, Massachusetts General Hospital, Boston, MA, USA
32 Neurology Department, Clínica Las Condes, Santiago, Chile

33 Department of Physical Medicine and Rehabilitation, Mayo Clinic, Rochester, MN, USA

34 Neurophysiology Department, Abbott Northwestern Hospital, Minneapolis, MN, USA

35 Clinical and Intraoperative Neurophysiology, Department of Neurosurgery, Hospital of University of Munich, Ludwig Maximilians Universitaet Muenchen (LMU), Munich, Germany

36 Clinical Neurophysiology Department, Hospital Español, Mexico City, Mexico

37 Intraoperative Monitoring Service, Mount Sinai West Hospital, New York, NY, USA

38 Intraoperative Neurophysiology Unit, Clinical Neurophysiology Department, Complejo Hospitalario de Navarra - B, Pamplona-Iruña, Navarra, Spain

39 Section of Neurosurgery, University of Manitoba, Winnipeg, MB, Canada 